\title{
CD138CAR-CD137/TCRzeta-expressing T Lymphocytes
}

National Cancer Institute

\section{Source}

National Cancer Institute. CD138CAR-CD137/T CRzeta-expressing T Lymphocytes. NCI

Thesaurus. Code C107505.

T-lymphocytes transduced with a retroviral vector expressing a chimeric antigen receptor (CAR) specific for syndecan-1 (CD138) (CART-138 T cells) coupled to the signaling domain of 4-1BB (CD137), and the zeta chain of the T-cell receptor (TCRzeta), with potential immunomodulating and antineoplastic activities. Upon transfusion, CD138CAR- CD137/T CRzeta -expressing T lymphocytes directs the T-lymphocytes to syndecan-1-expressing tumor cells and induces selective toxicity in those tumor cells. The 4-1BB co-stimulatory molecule signaling domain enhances activation and signaling after recognition of syndecan-1. Syndecan-1, a type 1 transmembrane proteoglycan and tumor associated antigen, is overexpressed in a variety of cancer cells. It plays a key role in the regulation of cell growth, differentiation, and adhesion, and its expression is correlated with poor prognosis. 